\title{
IMPACTO DE LAS FERIAS VOCACIONALES EN LA ORIENTACION VOCACIONAL
}

\section{Impact of the vocational fairs in the vocational orientation}

\author{
Margarita Olivares $P .{ }^{1} \&$ Jenny Quezada Z. ${ }^{2}$
}

\begin{abstract}
Resumen
En la presente investigación se busca determinar el impacto de las ferias vocacionales en la orientación vocacional. La muestra estuvo conformada por 215 estudiantes de sexo femenino, cursando el 5 to de secundaria y egresadas de secundaria, con edades entre los 15 y los 25 años, procedentes de centros educativos estatales y particulares de Lima. El instrumento utilizado fue un cuestionario de tipo opciones múltiples. Los resultados hallados son: El grupo de estudiantes evaluado, manifiesta que $82.8 \%$ tienen una decisión sobre que carrera profesional estudiar y un $17.5 \%$ no tiene dicha decisión. Las fuentes de información en orden de importancia que han utilizado para tomar su decisión vocacional son: el Internet, libros especializados y periódicos, revistas y las ferias vocacionales. Los criterios utilizados en orden jerárquico, en la toma de decisión de la profesión son: "la profesión que más le gustó", "el prestigio de la profesión" y "la recomendación de familiares y/o amigos". En relación al nivel de ayuda proporcionado por las ferias vocacionales para su toma de decisión, el $57.2 \%$ consideran que las ferias vocacionales aportaron poco en su decisión, mientras que el $36.7 \%$ opinan que fueron de marcada importancia en su elección profesional. En relación al grado de importancia que otorgan a los componentes presentados en las ferias vocacionales, las estudiantes le dan mayor importancia a la explicación oral (71.6\%), mientras que un $20.9 \%$ se interesó por la información escrita. Finalmente en relación a la posibilidad de poder considerar la conveniencia de la continuidad de las Ferias Vocacionales (FV), hallamos que el $91 \%$ opina a favor de seguir realizando estos eventos. El impacto de la FV en la orientación vocacional es parcial.
\end{abstract} Palabras clave: Orientación vocacional, ferias vocacionales.

\section{Summary}

The present investigation is to determine the impact of vocational fairs in vocational guidance. The sample consisted of 215 female students, enrolled in the 5th grade of secondary and high school graduates, aged 15 to 25 years, from state and private schools of Lima. The instrument used was one multiple-choice questionnaire. The results were: The group of students assessed, states that 82. 8\% have a decision on the career they want to study and $17.5 \%$ not have such a decision. The sources of information in order of importance they have used to take their career decision are: the Internet, specialized books and newspapers, magazines and vocational fairs. The criteria used in hierarchical order, in the decision making of the profession are "the most liked profession", "the prestige of the profession" and "the recommendation of family and I or friends." In relation to the level of assistance provided by vocational fairs for decision making, 57.2\% consider vocational fairs contributed little in his decision, while $36.7 \%$ think that were marked importance in their career choices. Regarding the degree of importance they attach to the components presented in vocational fairs, the students give more importance to the oral explanation (71.6\%), while $20.9 \%$ were interested in the written information. Finally in relation to the possibility to consider the advisability of continuing vocational fairs, we found that $91 \%$ believe in favor of continuing to make these events. The impact of the vocational fairs in the vocational orientation is partial.

Key words: vocational guidance/ vocational fairs

1. Psicóloga. Docente de la Facultad de Psicología de la Universidad Femenina del Sagrado Corazón.

2. Psicóloga. Docente de la Facultad de Psicología de la Universidad Femenina del Sagrado Corazón. 


\section{INTRODUCCIÓN}

Durante la juventud una de las decisiones más importantes que toma el joven adulto, es sobre los estudios a iniciar después de terminado el colegio secundario; es en este proceso que las instituciones educativas, aportan organizando ferias vocacionales, eventos en los cuales el estudiante de los últimos años de secundaria pueda obtener información sobre sus intereses vocacionales y las instituciones educativas de nivel superior.

Actualmente nos hallamos con una generación que ha nacido en una cultura tecnológica digital, se les ha llamado "nativos digitales", al respecto Joy, O (2013) p. 1, refiere:

El "nativo digital", un término acuñado por el autor estadounidense Marc Prensky en 2001, emerge como el grupo demográfico dominante en el mundo, mientras que el "inmigrante digital" se vuelve una reliquia de un momento previo. El concepto describe el cambio generacional en el que las personas son definidas por la cultura tecnológica con la que están familiarizadas. Prensky define a los nativos digitales como aquellos que nacieron en una "cultura nueva", mientras que los inmigrantes digitales son pobladores del viejo mundo, quienes vivieron en una era analógica e inmigraron al mundo digital y luchan más que los nativos para adaptarse al progreso de alta tecnología. Joy, O (2013)

\section{LAS FERIAS VOCACIONALES}

Una feria es definida como: Es un punto de encuentro entre la oferta que son los prestadores de servicios y productos y de la demanda que son los compradores o consumidores.

Una feria vocacional es la confluencia en un lugar y momento determinado de las instituciones educacionales, que cuentan con planes de formación académica, para los estudiantes que egresan de la enseñanza media, las que sumistran información de promoción y difusión acerca de las opciones de estudios que ofrecen mallas curriculares, duración y costo de las carreras, plana docente, infraestructura, alternativas de financiamiento, becas, campo laboral y otras variables a considerar, en un proceso de toma de decisión para la elección de una carrera profesional.
Para la presente investigación asumimos a las ferias vocacionales como lugares de encuentro entre las diferentes instituciones educativas y los estudiantes de educación secundaria, profesionales y otras personas interesadas en seguir estudios universitarios. Dichos eventos son auspiciados por instituciones educativas y/o empresas, en ellas se proporciona información oral y escrita sobre las modalidades de admisión, carreras profesionales que se ofrecen, duración de las carreras, costo de ellas, campo laboral, así como posibilidades de becas que ofrece la institución.

Los objetivos de las ferias vocacionales son:

- Dar a conocer las diferentes instituciones educativas.

- Que se conozcan las alternativas que ofrece el sistema educativo a nivel superior.

- Dar a conocer el perfil de las carreras profesionales.

- Proporcionar información sobre las diferentes modalidades de ingreso y de estudios.

- Orientar a los estudiantes con respecto a las interrogantes que estos presenten.

- Que el estudiante pueda comparar lo que las diferentes instituciones educativas le ofrecen.

- Se señalan los beneficios que implican el estudiar en la institución educativa superior.

Condiciones indispensables para la realización éxitosa de una feria vocacional:

- Disponer de personal que tenga una adecuada capacitación respecto a la oferta educativa que la institución ofrece.

- Contar con un espacio físico adecuado a fin de atender a los estudiantes y brindarles la información requerida.

- Brindar información directa sobre las carreras que se ofrecen a través de una exposición apoyada en material gráfico (folleteria) y audiovisual.

Características de las ferias vocacionales:

- Ubicación de las ferias

- En aulas: los alumnos pueden ingresar voluntariamente de acuerdo a sus intereses o 
el colegio organiza grupos de alumnos para que recepcionen la información, siendo el promedio de tiempo de la charla entre $20 \mathrm{y}$ 25 minutos.

- En el local del colegio: en el auditorio o en construcciones creadas en los espacios abiertos, en donde los alumnos concurren voluntariamente al stand de la institución educativa superior de su interés.

- En centros de convenciones.

- En el campus de la universidad.

- Duración de las ferias

- Horarios cortos: charlas vocacionales.

- Horarios fijos establecidos por la institución educativa.

- Horarios extendidos: puede ser una sola fecha o varias fechas, todo el día.

- Personal a cargo del evento

- Organizadores del evento: encargados de los departamentos de psicología, directores o subdirectores, encargados de carrera. y/o tutores.

- Participantes: instituciones educativas superiores representadas por las oficinas de admisión, quienes son las encargadas de organizar la participación de la institución, pudiendo concurrir a ellas: psicólogos, así como profesionales de diferentes áreas académicas, y /o alumnas capacitadas para proporcionar la información requerida.

- Material de difusión

- Materiales escritos: plegables, dipticos, trípticos, gigantografías, indicando carreras y modalidades de admisión que se ofrecen.

- Videos informativos.

- Material promocional

- Participantes de las ferias:

- Estudiantes de la propia institución educativa.

- Estudiantes de instituciones educativas afines geográfica o institucionalmente.

- Estudiantes y padres de familia de la propia institución y/o de las instituciones invitadas.

- Abiertas a la comunidad.
Las Ferias Vocacionales pueden complementarse con charlas que sean del interes del alumnado y sirvan para efectuar una adecuada elección vocacional.

La Universidad Femenina del Sagrado Corazón como institución educativa, viene participando desde la aparición de estos eventos en la década de los años 80 hasta la actualidad, caracterizándose por proporcionar en ellas, charlas de orientación vocacional e información institucional en localidades de Lima y otros departamentos.

Es a partir de estas experiencias que surgió la interrogante sobre el impacto de las ferias vocacionales en la elección de las carreras, planteándonos los siguientes objetivos:

\section{OBJETIVOS GENERALES}

Determinar el impacto de las ferias vocacionales en la orientación vocacional, tomando en cuenta la información, los criterios utilizados para la toma de decisión y el papel de las ferias vocacionales.

\section{OBJETIVOS ESPECÍFICOS}

1. Determinar las fuentes de información que utilizaron las estudiantes para tomar su decisión vocacional.

2. Determinar el orden jerárquico de los criterios utilizados en la toma de decisión de la profesión elegida,

3. Determinar la opinión de las estudiantes con respecto a la ayuda de las ferias vocacionales en su decisión

4. Determinar la opinión sobre la calidad de la información sobre profesiones, recibido a través de las ferias vocacionales

5. Determinar la opinión sobre el impacto de las ferias vocacionales en la decisión vocacional de los jóvenes

\section{Muestra}

La muestra estuvo conformada por 215 estudiantes de sexo femenino, cursando el 5to de secundaria y egresadas de secundaria, con edades entre los 15 y los 25 años, de las cuales $68 \%$ entre 15 y 16 años y $27.5 \%$ entre 17 y 18 años, procedentes de centros educativos estatales $(74 \%)$ y particulares $(26 \%)$ participantes a ferias vocacionales. 
Tabla 1 Distribución de la muestra de acuerdo a edad

\begin{tabular}{rcc}
\hline \multicolumn{1}{c}{ Edad } & Frecuencia & Porcentaje \\
\hline $15-16$ & 147 & 68.3 \\
$17-18$ & 59 & 27.5 \\
$19-20$ & 4 & 1.9 \\
+ de 21 & 5 & 2.4 \\
Total & 215 & 100 \\
\hline
\end{tabular}

\section{Instrumento}

El instrumento utilizado fue un cuestionario de tipo opciones múltiples, con un total de 11 reactivos, distribuidos de la manera:

- Información: (2 ítems).

- Toma de Decisión: (2 ítems).

- Papel de las Ferias Vocacionales: (7 items).

\section{Cuadro de nivel de impacto de las ferias vocacionales (FC)en la decisión vocacional}

\begin{tabular}{|c|c|c|c|}
\hline Indicador & Impacto total & Impacto parcial & No impacto \\
\hline $\begin{array}{l}\text { (FC) como fuente de } \\
\text { información para tomar } \\
\text { decisión vocacional }\end{array}$ & $\begin{array}{l}\text { (FC) en los primeros } 3 \\
\text { lugares. }\end{array}$ & (FC) en 4to o 5to lugar. & $\begin{array}{l}\text { (FC) no estar como fuente } \\
\text { de información. }\end{array}$ \\
\hline $\begin{array}{l}\text { Nivel de ayuda de las ferias } \\
\text { vocacionales }\end{array}$ & $\begin{array}{l}\text { (FC) ayuda de Marcada } \\
\text { Importancia }\end{array}$ & $\begin{array}{l}\text { (FC) ayuda de Poca } \\
\text { Importancia }\end{array}$ & $\begin{array}{l}\text { (FC) ayuda Nada de } \\
\text { Importancia }\end{array}$ \\
\hline Participación en las Ferias & $\begin{array}{l}\text { Más del } 75 \% \text { asistencia } \\
\text { voluntaria }\end{array}$ & Participación mixta. & Participación obligatoria. \\
\hline Usos del material & Usos prácticos & Compartir el material & $\begin{array}{l}\text { Material desechado o } \\
\text { guardado sin uso. }\end{array}$ \\
\hline $\begin{array}{l}\text { Elementos del material } \\
\text { considerados importantes }\end{array}$ & $\begin{array}{l}\text { Dos (2) elementos } \\
\text { considerados importantes }\end{array}$ & $\begin{array}{l}\text { Un (1) elemento } \\
\text { considerado importante }\end{array}$ & $\begin{array}{l}\text { Ningún elemento } \\
\text { considerado importante }\end{array}$ \\
\hline $\begin{array}{l}\text { Respuesta a una demanda } \\
\text { existente }\end{array}$ & $\begin{array}{l}\text { Responde a demanda sobre } \\
\text { información de carreras. }\end{array}$ & $\begin{array}{l}\text { Responde a demanda sobre } \\
\text { universidades del país. }\end{array}$ & $\begin{array}{l}\text { No responde a ninguna } \\
\text { demanda. }\end{array}$ \\
\hline $\begin{array}{l}\text { Nivel de importancia de las } \\
\text { ferias }\end{array}$ & Muy importante & Más o menos importante & Bajo nivel de importancia \\
\hline Calidad de la información & Calidad Alta & Calidad Promedio & Calidad Baja \\
\hline $\begin{array}{l}\text { Opinión sobre futuro de las } \\
\text { ferias }\end{array}$ & $\begin{array}{l}\text { Más del } 75 \% \text { a favor de } \\
\text { seguir realizándolas }\end{array}$ & $\begin{array}{l}\text { Menos del } 50 \% \text { a favor de } \\
\text { seguir realizándolas. }\end{array}$ & $\begin{array}{l}\text { Más del } 50 \% \text { rechazo a } \\
\text { seguir realizándolas. }\end{array}$ \\
\hline
\end{tabular}




\section{Resultados y discusión de resultados}

\section{INFORMACIÓN}

El grupo de estudiantes evaluado, manifiesta que en el momento de participar en la Feria Vocacional, el $82.8 \%$ si tenia decisión sobre que carrera profesional estudiar y un $17.5 \%$ no tenía aún la dicha decisión. En nuestra experiencia dicha decisión puede ser posteriormente modificada, especialmente durante los dos primeros años de estudios universitarios, ya sea por motivos de cambio de actitud hacia las tareas propias de la carrera y/o por motivos familiares.

Los motivos más frecuentes por los cuales el grupo de estudiantes que aún no tenían dicha decisión vocacional no habían efectuado su elección vocacional fueron: $17.7 \%$ porque le interesaban varias carreras a la vez y $7 \%$ porque les faltaba información para tomar la decisión.

Con respecto a las fuentes de información consideradas más importantes que han utilizado para tomar su decisión vocacional, fueron en primer lugar Internet, libros especializados y periódicos y revista, en segundo lugar y cuarto lugar las ferias vocacionales, en tercer lugar aparece nuevamente Internet, y en quinto lugar las charlas de orientación vocacional y el servicio de psicología del colegio.

De acuerdo a dichos resultados, no se comprobó la hipótesis de que las Ferias Vocacionales y los Servicios de Psicología ocupaban los primeros lugares como fuentes de información que utilizaron las estudiantes para tomar su decisión vocacional.

\section{Tabla 3. Porcentajes del Orden Jerárquico de las fuentes de información para tomar decisión vocacional}

\begin{tabular}{lccccc}
\hline $\begin{array}{l}\text { Fuentes de } \\
\text { información }\end{array}$ & $\begin{array}{c}\text { Primer } \\
\text { lugar }\end{array}$ & $\begin{array}{c}\text { Segundo } \\
\text { lugar }\end{array}$ & $\begin{array}{c}\text { Tercer } \\
\text { lugar }\end{array}$ & $\begin{array}{c}\text { Cuarto } \\
\text { lugar }\end{array}$ & $\begin{array}{c}\text { Quinto } \\
\text { lugar }\end{array}$ \\
\hline $\begin{array}{l}\text { Charlas de Orientación } \\
\text { vocacional }\end{array}$ & $11.6 \%$ & $10.7 \%$ & $8.4 \%$ & $14.4 \%$ & $37.7 \%$ \\
$\begin{array}{l}\text { Internet } \\
\text { Servicio de Psicología }\end{array}$ & $20.5 \%$ & $14.4 \%$ & $19.1 \%$ & $10.7 \%$ & $14.9 \%$ \\
del colegio & $16.3 \%$ & $12.6 \%$ & $10.7 \%$ & $10.2 \%$ & $19.5 \%$ \\
$\begin{array}{l}\text { Ferias Vocacionales } \\
\text { Libros especializados }\end{array}$ & $12.1 \%$ & $15.3 \%$ & $16.3 \%$ & $18.1 \%$ & $15.8 \%$ \\
Periódicos y revistas & $19.5 \%$ & $13.5 \%$ & $16.3 \%$ & $9.8 \%$ & $14.4 \%$ \\
& $18.6 \%$ & $14.4 \%$ & $13 \%$ & $14 \%$ & $13 \%$ \\
\hline
\end{tabular}

\section{TOMA DE DECISIÓN}

Con respecto a los criterios utilizados en la toma de decisión de la profesión son los siguientes: en Primer lugar (34\%), "la profesión que más le gustó", en Segundo lugar (19\%) "el prestigio de la profesión", en Tercer lugar "la "recomendación de familiares y/ o amigos" (20\%). Dichos criterios se relacionan con aspectos afectivos, o emocionales, de índole familiar y/o social.

De acuerdo a dichos resultados, no se comprobó la hipótesis de que los criterios de aspectos económicos y tiempo de estudios son los de mayor preferencia. 
Tabla 4. Porcentajes del Orden Jerárquico de de los criterios utilizados para tomar decisión vocacional

\begin{tabular}{lccccc}
\hline Criterios utilizados & $\begin{array}{c}\text { Primer } \\
\text { lugar }\end{array}$ & $\begin{array}{c}\text { Segundo } \\
\text { lugar }\end{array}$ & $\begin{array}{c}\text { Tercer } \\
\text { lugar }\end{array}$ & $\begin{array}{c}\text { Cuarto } \\
\text { lugar }\end{array}$ & $\begin{array}{c}\text { Quinto } \\
\text { lugar }\end{array}$ \\
\hline Prestigio de la profesión & $7 \%$ & $19.5 \%$ & $18.1 \%$ & $21.4 \%$ & $13.5 \%$ \\
Tiempo de estudios & $15.3 \%$ & $17.2 \%$ & $19.5 \%$ & $18.1 \%$ & $9.3 \%$ \\
Recomendación de familiares y/o amigos & $11.2 \%$ & $15.8 \%$ & $20.5 \%$ & $15.8 \%$ & $13.5 \%$ \\
Aspectos económicos & $12.6 \%$ & $17.2 \%$ & $14.4 \%$ & $19.1 \%$ & $15.8 \%$ \\
La profesión que más le gusto & $34 \%$ & $6.5 \%$ & $3.7 \%$ & $6.5 \%$ & $32.6 \%$ \\
& & & & & \\
\hline
\end{tabular}

En relación al nivel de ayuda proporcionado por las ferias vocacionales para su toma de decisión, el 57.2 $\%$ consideran que las ferias vocacionales aportaron poco en su decisión, mientras que el $36.7 \%$ opinan que fueron de marcada importancia en su elección profesional.

Tabla 5. Porcentajes de grado de aporte a toma de decisión vocacional de las Ferias vocacionales

\begin{tabular}{lcc}
\hline $\begin{array}{l}\text { Grado de } \\
\text { aporte }\end{array}$ & Frecuencia & Porcentaje \\
\hline Marcada Importancia & 79 & 36.7 \\
Poca Importancia & 123 & 57.2 \\
No importante & 13 & 6.0 \\
Total & 215 & 100 \\
\hline
\end{tabular}

\section{PAPEL DE LAS FERIAS VOCACIONALES}

La asistencia a las Ferias Vocacionales es propiciada por las direcciones educativas y los servicios de psicología de los centros educativos.

Con respecto a su asistencia a este tipo de eventos, un $84.2 \%$ de las encuestadas, informan que su participación ha sido voluntaria, mientras que el $12.6 \%$ indican que ha sido mixta.

Tabla 6. Porcentajes de tipo de asistencia a las Ferias vocacionales

\begin{tabular}{lcc}
\hline $\begin{array}{l}\text { Grado de } \\
\text { aporte }\end{array}$ & Frecuencia & Porcentaje \\
\hline Obligatoria & 7 & 3.3 \\
Voluntaria & 181 & 84.2 \\
Mixta & 27 & 12.6 \\
Total & 215 & 100 \\
\hline
\end{tabular}

En términos prácticos nos interesaba conocer el destino final de la folletería que se reparte en las ferias vocacionales, hallando que las actividades más frecuentes son Utilizarla con la familia $48.8 \%$, Seleccionar lo que le interesa $40 \%$ y Leerla $36.7 \%$. Al respecto es importante señalar el papel de la familia, como el grupo que en muchos casos influirá de manera decisiva en la toma de decisión. 
Tabla 7 Acción posterior realizada con la folleteria recibida en las Ferias vocacionales

\begin{tabular}{lcc}
\hline Acción & Frecuencia & Porcentaje \\
\hline Leerla & 79 & 36.7 \\
Desecharla & 6 & 2.8 \\
Seleccionar lo que le interesa & 86 & 40.0 \\
Utilizarlas con la familia & 105 & 48.8 \\
Compartirla con amigos & 30 & 14.0 \\
Guardarla sin uso & 10 & 4.7 \\
\hline
\end{tabular}

En relación al grado de importancia que otorgan a los componentes presentados en las ferias vocacionales, se indicará que las estudiantes le dan mayor importancia a la explicación oral (71.6\%), mientras que un $20.9 \%$ se interesó por la información escrita, solo un $7.4 \%$ le dio importancia a los afiches.

Tabla 8. Porcentajes de importancia del tipo de componente presentados en las Ferias vocacionales

\begin{tabular}{lcc}
\hline Tipo de Componente & Frecuencia & Porcentaje \\
\hline Explicación oral & 154 & 71.6 \\
Información escrita & 45 & 20.9 \\
Afiche & 16 & 7.4 \\
Total & 15 & 100 \\
\hline
\end{tabular}

Con respecto a los objetivos más importantes que a criterio de las alumnas debe cumplir una feria vocacional, hallamos en primer lugar la demanda de Información sobre las carreras $(88 \%)$, seguido por la necesidad de información sobre las universidades existentes en el país (64.2\%), convenios institucionales (63.3\%), información de modalidades de admisión $(61.4 \%)$ e información económica $(60.9 \%)$
Tabla 9. Orden de importancia de los objetivos de las Ferias vocacionales

\begin{tabular}{lcc}
\hline $\begin{array}{l}\text { Objetivos de } \\
\text { Feria vocacional }\end{array}$ & Frecuencia & Porcentaje \\
\hline Informar carreras & 190 & 88.4 \\
Conocer universidades & 138 & 64.2 \\
Conocer convenios institucionales. & 136 & 63.3 \\
Informar modalidades de admisión. & 132 & 61.4 \\
Información económica & 131 & 60.9 \\
Todas las anteriores & 121 & 56.3 \\
\hline
\end{tabular}

Respecto a la importancia que ha tenido para la elección profesional las ferias vocacionales, un $54 \%$ considera que ha sido Más o menos importante, mientras que un $36 \%$ le da gran importancia.

Tabla 10. Distribución porcentual de la opinión de importancia de las Ferias vocacionales

\begin{tabular}{lcc}
\hline $\begin{array}{l}\text { Grado de } \\
\text { importancia }\end{array}$ & Frecuencia & Porcentaje \\
\hline Poco & 20 & 9.3 \\
Más o menos & 117 & 54.4 \\
Muy importante & 78 & 36.3 \\
Total & 215 & 100 \\
\hline
\end{tabular}

En referencia a la calidad de información sobre las profesiones, recibida en las Ferias vocacionales, hallamos que un $48 \%$ opina que dicha calidad es Promedio, sin embargo el $41 \%$ opina que el nivel de información recibida es Alta. 
Tabla 11. Porcentajes de la opinión de la calidad de la información de las Ferias vocacionales

\begin{tabular}{lcc}
\hline $\begin{array}{l}\text { Grado de } \\
\text { calidad }\end{array}$ & Frecuencia & Porcentaje \\
\hline Baja & 7 & 3.3 \\
Promedio & 104 & 48.4 \\
Alta & 88 & 40.9 \\
Total & 215 & 100 \\
\hline
\end{tabular}

Finalmente en relación a la posibilidad de poder considerar la conveniencia de la continuidad de las Ferias vocacionales, hallamos que el $91 \%$ opina a favor de seguir realizando estos eventos. Las ferias les permite a las estudiantes tener la información reunida, comparar la información, constatar si aquello que han leído en otros medios es veraz.

\section{Tabla 12. Porcentaje de Opinión a favor o en} contra de las Ferias vocacionales

\begin{tabular}{lcc}
\hline Opinión & Frecuencia & Porcentaje \\
\hline A favor & 195 & 90.7 \\
En contra & 12 & 5.6 \\
No contestaron & 8 & 3.7 \\
Total & 215 & 100 \\
\hline
\end{tabular}

Considerando que las Ferias Vocacionales (FV) se hallan como fuente de información en los dos primeros lugares, un $30.7 \%$ considera que el nivel de ayuda de las ferias vocacionales son de Marcada Importancia, el $84 \%$ participa de manera voluntaria, al material de folleteria se le da un uso práctico en un 59\%, se resalta los elementos de explicación oral como importante en un $72 \%$; las FV responden a una demanda de información sobre las carreras; el 36\% considera Muy importante las FC, la calidad de la información es de un nivel Alto de acuerdo al $41 \%$ y hay una opinión a Favor de seguir realizando las FV en un $91 \%$., se concluye que el nivel de impacto de las Ferias Vocacionales en la orientación vocacional es parcial.

\section{CONCLUSIONES}

1. Las fuentes de información consideradas más importantes que han utilizado para tomar su decisión vocacional, fueron en primer lugar Internet, libros especializados y periódicos y revista, en segundo lugar y cuarto lugar las ferias vocacionales, en tercer lugar aparece nuevamente Internet, y en quinto lugar las charlas de orientación vocacional y el servicio de psicología del colegio.

2. Los criterios utilizados en la toma de decisión de la profesión son los siguientes: en Primer lugar (34\%), "la profesión que más le gustó", en Segundo lugar (19\%) "el prestigio de la profesión", en Tercer lugar "la "recomendación de familiares y/o amigos" (20\%).

3. El nivel de ayuda proporcionado por las ferias vocacionales para su toma de decisión, el 57.2 $\%$ consideran que las ferias vocacionales aportaron poco en su decisión, mientras que el $36.7 \%$ opinan que fueron de marcada importancia en su elección profesional.

4. Con respecto a la calidad de información sobre las profesiones, recibida en las Ferias vocacionales, hallamos que un $48 \%$ opina que dicha calidad es Promedio, sin embargo el $41 \%$ opina que el nivel de información recibida es Alta.

5. Los objetivos más importantes que a criterio de las alumnas deben cumplir las ferias vocacionales, hallamos en primer lugar la demanda de Información sobre las carreras (88\%), seguido porlanecesidad deinformación sobre las universidades existentes en el país, convenios institucionales, información de modalidades de admisión e información económica. 
6. Respecto a la importancia que ha tenido para la elección profesional las ferias vocacionales, un $54 \%$ considera que ha sido Más o menos importante, mientras que un $36 \%$ le da gran importancia.

7. La opinión de continuar la realización de las Ferias Vocacionales, es Favorable en el 91\%.

8. El impacto de las Ferias vocacionales en la orientación vocacional es de un nivel de Impacto Total.

\section{REFERENCIAS}

Joy Oliver (2013) Nativos digitales: ¿Quiénes son y qué significa?. Publicado el 13 de enero 2013. Disponible en: http://cnnespanol.cnn. com/2013/01/25/nativos-digitales-quienes-son-yque-significa/
Feixa Carlex GENERACIÓN @ LA JUVENTUD EN LA ERA DIGITAL NÓMADAS 76- $91 \mathrm{http} / / /$ www.ucentral.edu.co/movil/images/stories/iesco/ revista_nomadas/13/nomadas_13_6_generacion. PDF

Feixa Carlex (2006) Generación XX. Teorías sobre la juventud en la era contemporánea Revista Latinoamericana de Ciencias Sociales, Niñez y Juventud. 4 (2). Disponible en: http:// www.s/cpoliticas/file.php/35/manuel_torres/ Feixa__Generacion_XX_Teorias_juventud_ contemporaneas.pdf

Ministerio de Educación (2011) Tutoría y Orientación educativa. Orientación vocacional. Disponible en: http://ditoe.minedu.gob.pe/ Materiales\%20DITOE/COV.pdf

Universia (2012) Orientación vocacional Disponible en: http://pre.universia.edu.pe/que-estudiar/ orientacion-vocacional/

Fecha de recepción: 20 de mayo 2013

Fecha de aceptación: 6 de junio 2013 